\title{
Same and Different in Trans-Local East Asian Medicine
}

\author{
Judith Farquhar
}

Published online: 21 December 2012

(C) Springer Science+Business Media New York 2012

Why should medical anthropologists be interested in East Asian traditional medicine? Especially, we might ask, why should anyone-clinician or anthropologist-care about the history of the abstruse knowledge and highly technical practice that have been "traditional" in China, Japan, and Korea? The four papers that are gathered in this special issue of Culture, Medicine, and Psychiatry, along with a thoughtful introduction by issue editor Volker Scheid, provide a number of answers to these questions. Even as each article engages in detail with a particular rich strain of discourse and healing in a particular time and place in East Asia, all of them provide resources for thinking far beyond Asia and even far beyond medicine. Following the lead of these scholars, we can let the concepts and terminologies of Asian medical systems open up new aspects of the diverse lived worlds that have always interested us as anthropologists.

For one thing, studying the various medicines of the world allows us to ask just how diverse, how deeply variable, lived worlds have been. When can we presume that bodies on different continents are the same, or hypothesize that some diseases are entirely local? What was "miasma" or "soul-loss" in the experience of our ancestors, and thus, how were their "bodies" different? These are important questions to address to medicine because every "medical system" comes into play, historically, in situations where much is at stake. Sufferers and their healers, as they address disorder and pathology, are not merely entertaining themselves with charming metaphors, persuasive interpretations, or culturally appropriate interaction styles. They are engaging with real forces that intervene in human being itself, and this engagement is not always pretty or gentle. (See, for example, the far from kindly medical advice of Wada Tokaku cited in Keiko Daidoji's paper, a bracing blast of common sense from eighteenth century Japan. One could also note the iatrogenic discomforts of Asian techniques of scraping, cupping, and needling, and

\footnotetext{
J. Farquhar $(\bowtie)$

Department of Anthropology, University of Chicago, Chicago, USA

e-mail: farquhar@uchicago.edu
} 
the horrid "side effects" of chemotherapy.) Yet recognized pathological forces may not be universally at work on identically structured bodies; we must entertain the possibility that reality is multiple, materially and in depth. As Shigehisa Kuriyama (1999) asks in The Expressiveness of the Body, if medicine everywhere has had to deal with a single "nature," or the "same body," how is it that medical language, ideas, and imaginaries have varied so much, and been so deeply incommensurate? The diversities of medical action and bodily being in history thus pose one of anthropology's fundamental questions: how deep does difference go? And the archives of East Asian medicine offer fabulous resources for our efforts to answer it.

There is no shortage of incommensurabilities or deep ontological differences in the papers gathered in this issue. The physiological fire of Soyoung Suh's hwa-byung is an example, along with the very widespread experience of han anger, stress, and regret noted by psychiatrists working in post-war Korea. Karchmer's and Scheid's "Liver" and "Heart," being visceral systems of function rather than anatomical organs, though they are named by familiar names, also appear quite untranslatable (though they certainly travel). Pathologies named with reference to "dampness" and "phlegm" (see Karchmer 2005) simply do not map over biomedical nosologies, despite many modern efforts to produce correspondence tables between Chinese and Western medical systems. And the "inner poison" that Daidoji tells us worried doctors in early twentieth century Japan is no ordinary toxin but a thoroughly situated, socially complex illness factor. Historical and epistemological science studies, sometimes thinking of such hard-to-translate things as boundary objects (Bowker and Star 1999), have begun to demonstrate how attempts to commensurate categories, or compare ways of carving up reality, reflect differing lived worlds. Social studies of medicine have shown how, in encounters between world medicines, skilled practitioners struggle to delimit separate spheres of authority, based on quite different views of how natural processes work. These are not just differences in concepts or representations; the stubborn materiality of medical/bodily entities cannot be dissolved by intercultural conversation or agreement on (translated) terms.

The papers in this issue constitute a substantial contribution to the deep historicization of objects of medical interest: diseases or named disorders (depression, neurasthenia, Liver qi constraint), standardized drug formulas (Rambling Powder), body parts (Livers, Hearts, even Minds), and, of course, qi. Once the emergence and deployment of these things has been carefully tracked, we can no longer see any medicine as directly addressing "Nature" as nature has been rendered in modernist thought. Though Volker Scheid in his Introduction to this issue baldly states that medical people everywhere deal with "what is, after all, the same body," this appears to be more an article of faith than a historical fact discoverable through even his own research. Instead, historical investigation keeps giving us medicines that work with "fundamentally different conceptions of the body/person" and show historical trajectories - or "currents"-that extend through time and attract doctors, patients, and thinkers almost regardless of clinical effectiveness.

It is worth noting that this sort of project banishes the diagnosis, found in an earlier medical anthropology, of "culture-bound syndromes." Culture-bound 
syndromes like the classical koro and amok phenomena (just to stick to Asia) must now be taken seriously in light of the actual things and forces they manifest or embody. No more denunciations of mass hysteria allowed. The bizarre complaints and behavioral deviations of (implicitly primitive and confused) others no longer stand as a "cultural" and local contrast to the "natural" and universal diseases named by biomedicine. At the same time, as Scheid notes when he opens his Introduction by declaring depression to be a culture-bound syndrome, the approach taken by these papers gives us a new hypothesis to bring to medical anthropology: to what extent, and why, is any disorder that interests us "culture bound"? What is the culture in question? What is the nature of the constraints that bind a pattern of symptoms to a wider world of cultural practice?

These questions, of course, become ever more salient as anthropology turns to understanding the new spaces, times, and trajectories of globalization. Daidoji, Karchmer, Scheid and Suh all agree that the various conditions they study have achieved some form of trans-locality, and they do a wonderful job of tracing the currents that have carried transforming syndromes and treatments through (Asian and global) time and space. East Asian medicine, with all its differences and conflations, now thrives in clinics close to all our homes. But this de facto translation of a medicine of qi-transformation into communities for whom qi does not exist does not put an end to metaphysical debates, far from it. These authors write - as we all do-in a world of "global health" and "translational medicine" where both medical interventions and historical accounts must be rendered legitimate in relation to "evidence." Though one could argue that objective evidence really functions as a "base" for no medicine (and the vigorous activity now going on to insert evidence at the foundations of medical decision-making, with a great sense of urgency, attests to this), we nevertheless see a tremendous cultural anxiety as health care programs spread around the world, biomedicine claiming a privileged healing authority and the right to triage access to services by sufferers (Nguyen 2010). How have readers and users of medical heritage in East Asia assessed the question of authority and evidence? How have they tried to deal with the frictions, gaps, and interruptions of several kinds of objectivity and rationality in the systems of medicine they know and use?

One answer to this question, of course, is that they have dealt with medical heterogeneity in practice (see especially Daidoji). As all these papers attest, Asian medicine in all its settings is always already "integrated" on the ground. TCM in the United States, for example, is far from being an entirely pure or separate "complement" or "alternative" to biomedicine; its very form, as Karchmer among others has shown (2005), incorporates certain colonial logics derived from an expansive world biomedicine. But in the actual integrative practical regimes we study, we discover a great many hybrid objects that are put into daily practice (think acupuncture meridians, Ayurvedic dosas, homeopathic dilutions). How do practitioners and sufferers - seeking, perhaps, an evidence base-satisfy themselves that their effective (or even not yet effective) treatments are "in the true," to adopt Canguilhem's and Foucault's famous phrase? One could also ask, do they need to ask? Or are they content with ad hoc successes, good clinical judgment on the fly? 
I'm not sure. I was recently talking with an herbalist with a considerable local reputation in China's Guangxi Province about the "theoretical foundations" of his work. He said he most relied on the divinatory logic of the Book of Changes. Aware that divination has often been denounced in China as superstition, he added, "I don't know if this stuff is true or not, maybe it's just superstition; but I know that it works." Even as he shows himself to be a rural intellectual steeped in China's textual tradition, this village doctor leads us away from epistemological questions to practical ones. Or perhaps he encourages us to consider what kind of knowing is most salient in medical practice.

In tune with this orientation, then, I'd like to think away from the "problem" of differences and ask whether some kinds of sameness might also inform a trans-local anthropology of medicine - historically, epistemologically, clinically, experientially. Though these authors don't highlight this particular aim and effect of their writing, with the diagnosis of constraint, the papers in this issue offer us an enticing common ground. We can read these translated and paraphrased visions from doctors in China, Japan, and Korea as analytical anthropologists, engaging with contradictory realities to make richer comparisons and achieve more thorough epistemological resolutions. But we can also read as embodied people who are not strangers to discomfort, stress, alienation, anxiety, unhappiness, victimization, trauma. "Depression," after all, is not a purely mental experience. When I think about it, I actually can't imagine what a purely mental experience would be like - could it be experience at all? When I have a good idea I breathe differently and my fingers itch for the keyboard; when I anticipate an upcoming showdown I perspire and stammer; feeling overwhelmed with work makes my back and shoulders hurt. I can more easily imagine a state of having weak nerves throughout my body, i.e., neurasthenia, than I can admit to suffering from depression. And it is even easier to feel constriction in the chest together with flashes of fire rising to my face and scalp, once we have a less (or differently) medicalized language for these symptoms. No wonder so many modern Koreans have hwa-byung! The "culture" that "binds" this syndrome to the lives of moderns is everywhere; it is difficult indeed to evade the effects of these constraints. And once we have learned as embodied anthropologists to think and feel constraint, it becomes a condition that inhabits - at least sometimes, for at least many of us - the everyday body-mind and life. As it does so, "mental illness" looks more and more like a culture-bound syndrome.

And what about the widely acknowledged materiality of emotions? All the authors are very careful to denaturalize and historicize the "seven emotions" and han anger, respectively. Even comparing modern China and South Korea yields big differences between ways of imagining the experience, effects, and dangers of emotional life. But both/all of these versions of feeling are, with a little effort, uncannily imaginable even for thoroughly Anglophone readers. In my own experience of observing doctors of TCM at work in China, for example, I have often heard them advise patients, "for your own good, don't 
get angry." (Yanhua Zhang 2007 has also noted and interpreted this common medical injunction, which seeks to protect patients from the ravages of an emotional force that can cause further physiological damage.) As an American, though, one is inclined to ask, how can I help getting angry? When I'm angry, the emotion takes over, doesn't it? Still, as I tried to imagine how I would prevent myself from getting angry, for the sake of my general health, I began to notice how angry most people are. I didn't like what I saw. As I got better at bracketing violent feelings, containing social vitriol, holding the foolishness of others at a bemused distance - in short, as I learned to "not get angry" - I also wondered more pointedly why so many of my friends and neighbors had never learned this skill. Why do we let ourselves be victimized by our emotions? Could the culture-bound yet bracketable forces of anger, fear, joy, sorrow, reflection, grief, and longing begin to make sense in and of our lives in North America?

These are questions asked more by the embodied reader than by the critical anthropologist, though I have not myself been good at separating these two functions. The critic would point out that phenomenological, participatory, appropriative comparison of this kind evades some key questions: what, for example, is the "self" that could refuse anger or moderate extreme joy? Moreover, what notion of health would be advanced by a bracketing of the emotions in order not to be victimized by them? There are so many differences to be accounted for, and any insistence on sameness risks self-indulgence, conservatism, romanticism, and exclusion of the culturally illegible.

Still, the articles in this special issue not only note but also participate in the trans-local travel of diverse real things. Scholar-doctors like Scheid and Karchmer encounter qi, constraint, the seven emotions, and the Liver in their clinics in London and North Carolina; cultural historians like Daidoji and Suh write of han, fire, neurasthenia and $k i$ in a non-metaphorical, realist language as they avoid "othering the past" (de Certeau 1988). The historiography these authors adopt, its agenda ultimately derived from contemporary practices, is instructive for those of us who would like to find theoretical riches in comparing "medical systems" and "culturebound syndromes." With studies like these, we can begin to think creatively about the depths at which human being has differed. But we can also take very personal and human pleasure in the discovery of experiences that must, we feel, be the same. When medical history is well and sensitively done, as these studies are, we readers are given imaginative access to old-new potentials in our own existence. Edo bodies, late-Qing and Republican Era bodies, modern Korean bodies-are all potentially our own lived bodies, providing we accept the pluralization achieved by histories of being, the multiplicity inherent to being itself.

\section{References}

Bowker, Geoffrey, and Susan Leigh Star

1999 Sorting Things Out: Classification and Its Consequences. Cambridge MA: MIT Press. 
de Certeau, Michel

1988 The Writing of History. New York: Columbia University Press.

Karchmer, Eric

2005 Orientalizing the Body: Postcolonial Transformations in Chinese Medicine. Ph.D. dissertation. University of North Carolina.

Kuriyama, Shigehisa

1999 The Expressiveness of the Body and the divergence of Greek and Chinese Medicine. New York: Zone Books.

Nguyen, Vinh Kim

2010 The Republic of Therapy: Triage and sovereignty in West Africa's Time of AIDS. Durham NC: Duke University Press.

Zhang, Yanhua

2007 Transforming Emotions with Chinese Medicine: An Ethnographic Account from Contemporary China. Albany: SUNY Press. 